\title{
MicroRNA-200c impairs uterine receptivity formation by targeting FUT4 and a1,3-fucosylation
}

\author{
Qin Zheng ${ }^{1,3}$, Dandan Zhang ${ }^{1,3}$, Yu Yang ${ }^{1}$, Xinyuan Cui ${ }^{1}$, Jiaqi Sun ${ }^{1}$, Caixia Liang ${ }^{1}$, Huamin Qin $^{2}$, Xuesong Yang ${ }^{1}$, Shuai Liu ${ }^{*, 1}$ and \\ Qiu Yan ${ }^{\star 1}$
}

Successful embryo implantation requires the establishment of a receptive endometrium. Poor endometrial receptivity has generally been considered as a major cause of infertility. Protein glycosylation is associated with many physiological and pathological processes. The fucosylation is catalyzed by the specific fucosyltransferases. Fucosyltransferase IV (FUT4) is the key enzyme for the biosynthesis of $\alpha 1,3-$ fucosylated glycans carried by glycoproteins, and the previous studies showed FUT4 expression changed dynamically during perimplantation. MicroRNAs (miRNAs) are known to regulate specific gene expression. However, the relationship between specific miRNA and FUT4, as well as the role of miRNA/FUT4 in the establishment of uterine receptivity remains elusive. In the current study, we reported that the levels of miR-200 family members were significantly increased in serum from infertility and abortion patients relative to healthy non-pregnancy and early-pregnancy women. Among these, miR-200c was the most sensitive diagnostic criterion for infertility by receiver operating characteristic curve analysis. FUT4 was lower in the serum from infertility and abortion patients compared with the healthy non-pregnancy and early-pregnancy women. Using endometrial cell lines and a mouse model, we demonstrated that miR-200c targeted and inhibited FUT4 expression, leading to the dysfunction of uterine receptivity. Our results also revealed that miR-200c decreased $\alpha 1.3$-fucosylation on glycoprotein CD44, which further inactivated Wnt/ $\beta$-catenin signaling pathway. Taken together, miR-200c hampers uterine receptivity formation by targeting FUT4 and $\alpha 1$.3-fucosylation on CD44. miR-200c and FUT4 may be applied together as the potential markers for endometrial receptivity, and useful diagnostic and therapeutic targets for infertility.

Cell Death and Differentiation (2017) 24, 2161-2172; doi:10.1038/cdd.2017.136; published online 15 September 2017

The essential events of a successful pregnancy requires the implantation of a competent blastocyst into a receptive endometrium. ${ }^{1}$ The uterine endometrium is only receptive to the blastocyst within a short period known as the 'window of implantation' (WOI). During this period, the endometrium undergoes characteristic morphological and molecular changes that allow embryo to adhere and invade, including an increasing pattern of microvilli of the epithelial cells and pinopode formation, as well as alterations in adhesive molecules and cytokines and so on. ${ }^{2-4}$ Abnormalities in molecular structures and expression often hinder the development of uterine receptivity, which induce reproductive disorders, such as infertility, abortion and preeclampsia. ${ }^{5-7}$ Despite advances in the understanding of reproductive processes, the mechanism of post-transcriptional regulation and implantation failure remains unclear in infertility and abortion.

MicroRNAs (miRNAs) are endogenous noncoding RNAs that function in the post-transcriptional regulation of gene expression by targeting specific mRNAs of the gene for degradation and translational repression. miRNAs have emerged as the major bioregulatory molecules of many physiological processes including reproduction. ${ }^{8}$ For the fetus, specific miRNAs are highly expressed in human embryonic cells, which regulate blastocyst activation, embryo implantation and development. ${ }^{9}$ For the mother, different kinds of miRNAs are expressed and secreted by the uterine endometrium during human menstrual cycle and embryo implantation that are associated with uterine receptivity formulation and stromal decidualization and so on. ${ }^{10,11}$ Recently, miRNA expression profiles have been described in several pathological gynecological conditions including infertility, endometriosis and preeclampsia. ${ }^{12-14}$ The miRNA-200 family (miR-200a, miR-200b, miR-200c, miR-141 and miR-429) arises from two gene clusters. Previous studies demonstrated that the expression of miR-200 family members was low in the receptive uterus. ${ }^{15}$ For example, the expression of miR-200a, miR-200b and miR-200c in mouse endometrial stromal cells was downregulated during implantation. ${ }^{16}$ Similarly, miR-200a, miR-200c, miR-141 and miR-429 were decreased in human decidualization model in vitro. ${ }^{17}$ However, very little information is available about the expression of miR-200 family members and their targets in infertility and abortion, especially the functions in uterine receptivity formation.

Protein glycosylation, a common post-translational modification, is involved in many physiological and pathological processes. The glycobiology of implantation shows that fucosylation of the uterine endometrium mediates recognition

\footnotetext{
${ }^{1}$ Department of Biochemistry and Molecular Biology, Dalian Medical University, Liaoning Provincial Core Lab of Glycobiology and Glycoengineering, Dalian 116044, China and ${ }^{2}$ Department of Pathology, the Secondary Affiliated Hospital of Dalian Medical University, Dalian 116000, People's Republic of China

${ }^{*}$ Corresponding author: S Liu or Q Yan, Department of Biochemistry and Molecular Biology, Dalian Medical University, Liaoning Provincial Core Lab of Glycobiology and Glycoengineering, Dalian 116044, China. Tel/Fax: +86 411 86110308; E-mail: liushuai_129@163.com or yanqiu63@126.com

${ }^{3}$ These authors contributed equally to this work

Received 11.5.17; revised 17.7.17; accepted 19.7.17; Edited by A Willis; published online 15.9.17
} 
a

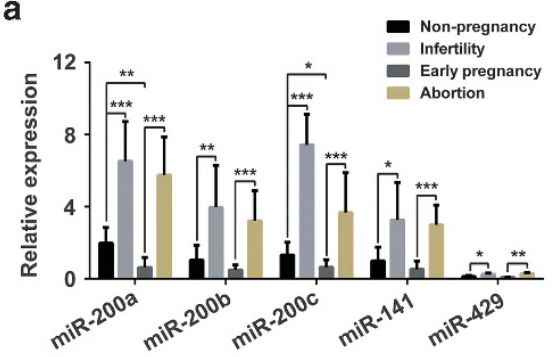

b

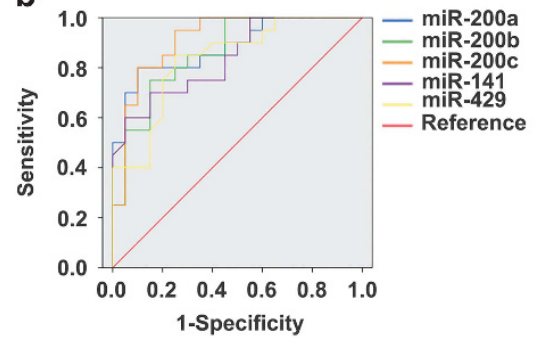

\begin{tabular}{lc}
\multicolumn{2}{c}{$\begin{array}{r}\text { ROC curve analysis of infertility } \\
\text { patients and healthy controls }\end{array}$} \\
\hline Variable & Area under the curve \\
\hline miR-200a & 0.883 \\
miR-200b & 0.860 \\
miR-200c & 0.913 \\
miR-141 & 0.839 \\
miR-429 & 0.834 \\
\hline
\end{tabular}

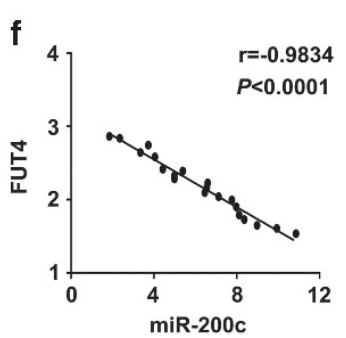

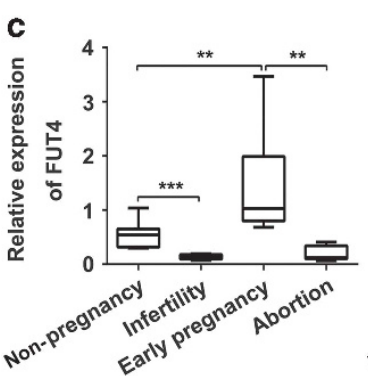

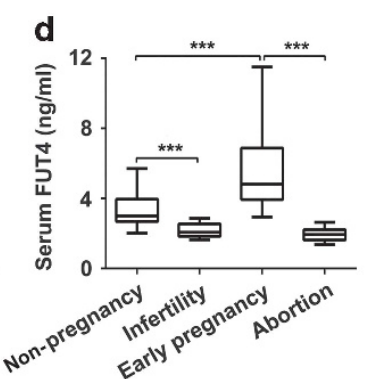

e

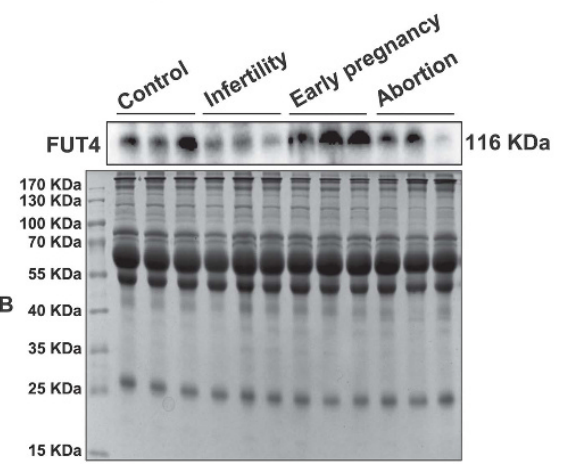

Figure 1 High miR-200 family and low FUT4 levels in infertility and abortion patients. (a) Serum levels of miR-200 family members in healthy control (Non-pregnancy), infertility, early pregnancy and abortion detected by real-time PCR. (b) ROC curve analysis of the diagnostic value of miR-200 family members for infertility. (c-e) Detection of FUT4 expression by real-time PCR (c), ELISA (d) and western blot (e). (f) Correlation analysis between miR-200c and FUT4 expression in serum of healthy control and infertility patients. ROC: receiver operating characteristic. CBB: coomassie brilliant blue. The statistical analysis was shown. ${ }^{\star} P<0.05,{ }^{* \star} P<0.01,{ }^{* \star \star} P<0.001$

and adhesion at the fetal-maternal interface, thereby contributing to a successful pregnancy. ${ }^{18,19}$ Fucosylated glycans carried by the glycoproteins are catalyzed by the specific fucosyltransferases (FUTs). FUT4 is an $a-1,3$ fucosyltransferase that adds the UDP-L-fucose to the scaffold glycoproteins to form $a-1,3$-linkage fucosylated glycans, such as Lewis $Y$ (LeY, Fuc a1-2 Gal- $\beta 1-4$ [Fuc a1-3]GlcNAc $\beta 1$ ), a difucosylated oligosaccharide. LeY and FUT4 on the uterine cells and tissues of different species change dynamically during preimplantation. LeY is significantly enriched on the surface of the uterine epithelium of human, monkey, rat and mouse during menstrual cycle and embryo implantation period. ${ }^{20-22}$ Ponnampalam et al. ${ }^{23}$ reported an increased expression of FUT4 in the secretory phase compared with that in the proliferative phase in human uterine endometrium. Our previous data showed that FUT4 expression was higher in receptive uterine epithelial cells. ${ }^{24}$ Here, we further aimed to find specific miRNA that targeted FUT4, and explore the role of specific miRNA /FUT4 in uterine receptivity formation.

Glycoproteins on cell surface are closely associated with cellular functions, such as cell recognition, adhesion and signal transduction. ${ }^{25}$ CD44 is a type I transmembrane glycoprotein. ${ }^{26}$ It has been demonstrated that CD44 is expressed intensely in the endometrial epithelium and on stromal during the mid- and late secretory phases; whereas the expression is not detectable during the proliferative phase in human. ${ }^{27,28}$ The diminished expression of CD44 in decidual cells caused spontaneous abortion, and the impaired development of early endometriotic lesions was found in CD44 knockout mice. ${ }^{29}$ Therefore, CD44 may function at an early stage of the adhesive contact between the endometrium and blastocyst. Specifically, altered expression of FUTs can change the fucosylation modification of glycoproteins to further influence the cellular functions. It is reported that CD44 is abundant in $a$-fucosylation, especially $a-1,2$ and $a-$ 1,3-linkage, in breast cancer cells. The increased LeY antigen strengthens CD44-meditated cell adhesion. ${ }^{30}$ However, the role of fucosylation of CD44 regulated by FUT4 during uterine receptivity formation remains unclear.

In the present study, we found that miR-200c in the serum of pregnant women was decreased compared with that healthy non-pregnancy women, and increased in the infertility and abortion patients. We demonstrated that FUT4 was the target of miR-200c, and miR-200c inhibited FUT4 and $a-1,3-$ fucosylation biosynthesis, particularly on CD44, thus hampering uterine receptivity and embryo implantation in vitro and in vivo.

\section{Results}

High miR-200 family members and low FUT4 levels in infertility and abortion patients. We first performed realtime PCR, ELISA and western blot to determine the levels of the miR-200 family members (miR-200a, miR-200b, miR-200c, miR-141 and miR-429) and FUT4 in the serum of the healthy control (non-pregnancy), infertility, early-pregnancy and abortion women. As shown in Figure 1, the levels of miR-200 family members was increased in the serum of infertility patients compared with the healthy non-pregnancy women. The levels of miR-200a and miR-200c were lower in early-pregnancy women than that in non-pregnancy control, whereas the levels of the miR-200 family members were higher in abortion patients than that in early-pregnancy 
women (Figure 1a). We further evaluated the diagnostic value of miR-200 family members by receiver operating characteristic curve (ROC) analysis, and determined miR-200c (area under the curve: 0.913 ) as the best decisive threshold among miR-200 family members (Figure $1 \mathrm{~b}$ ).

Based on the previous study in that FUT4 was highly expressed in the receptive uterus, we detected FUT4 expression among non-pregnancy, infertility, early-pregnancy and abortion women to analyze the variations in the human endometrium during different functional states (Figures 1c-e). The real-time PCR, ELISA and western blot analysis showed that FUT4 level was decreased in infertility patients, whereas increased in early-pregnancy women compared with the nonpregnancy control. The results also showed that FUT4 level was lower in abortion patients than that in early-pregnancy women. The above data implies the potential involvement of FUT4 in uterine receptivity formation. It is worthy noticing that there is a negative correlation between miR-200c and FUT4 expression levels $(r=-0.9834)$ in the serum of patients with infertility (Figure 1f).

miR-200c inhibits proliferation and receptive ability of uterine epithelial cells in vitro. Having observed the differential expression of miR-200 family members in human serum, and determined that miR-200c was the most sensitive and specific marker for infertility, we went on to functionally characterize miR-200c by focusing on its effect on proliferation and the receptive ability of the uterine epithelial cells. The results indicated that the level of miR-200c was increased after cells were transfected with miR-200c mimics, whereas decreased after Anti-miR-200c transfection in RL95-2 and Ishikawa cells by real-time PCR (Figures $2 a$ and f).

CCK-8 (Figures $2 \mathrm{~b}$ and $\mathrm{g}$ ) and colony formation assay (Figures $2 \mathrm{c}$ and $\mathrm{h}$ ) were performed to assess the effect of miR-200c on cell proliferation. RL95-2 and Ishikawa cells transfected with miR-200c mimic showed lower proliferative rate and fewer number of colonies than the control cells. In contrast, RL95-2 and Ishikawa cells transfected with AntimiR-200c exhibited higher proliferative rate and a greater number of colonies than that control cells.

We next investigated the potential role of miR-200c in modulating the receptivity formation of uterine epithelial cells. As shown in Figures $2 \mathrm{~d}$ and $\mathrm{i}$, an obvious morphological alteration in the ultrastructure of RL95-2 and Ishikawa cells was observed by scanning electron microscopy (SEM). Cells with higher miR-200c level displayed fewer microvilli on the cell surface, which is a marker of uterine receptivity. The embryo adhesion model in vitro also showed that RL95-2 and Ishikawa cells transfected with miR-200c mimics exhibited significantly reduced rates of adhesion to embryonic cells compared to the control cells, whereas increased adhesion rates in Anti-miR-200c transfection cells relative to the control cells (Figures 2e and j).

FUT4 is a novel target of miR-200c. To uncover the mechanism by which miR-200c affected cell proliferation and receptivity establishment in uterine epithelial cells, we made an attempt to identify potential target genes of miR-200c. Because our previous study showed that highly receptive uterine epithelial cells expressed a higher level of
FUT4 than lower receptive cells, as well as the negative correlation between miR-200c and FUT4, we then explored whether FUT4 was a new target of miR-200c. Over 10 databases, including TargetScan, PicTar and miRanda were searched for potential genes under the control of miR-200c. Among the target genes, FUT4 was a potential target of miR-200c with high matched base sequence between the 3'UTR of FUT4 and miR-200c (Figure 3a). To further verify whether miR-200c would bind directly to the 3'-UTR of FUT4, the wild-type (WT) 3'-UTR or mutant-type (MUT) 3'-UTR target sequences were cloned into the luciferase reporter vector (pGL3), and transfected into cells with a reference vector. The results showed that the luciferase activity of FUT4 was significantly decreased after co-transfection of the WT luciferase reporter construct and miR-200c mimics, whereas little changes were observed in enzymetic activity when cotransfection of MUT luciferase reporter construct and miR-200c mimics in the kinds of uterine epithelial cells (Figures $3 b$ and $h$ ). To evaluate the regulatory role played by miR-200c on FUT4 gene and protein expression, real-time PCR (Figures $3 c$ and i) and western blot (Figures $3 d-f$ and jI) were performed. The data showed that miR-200c mimics transfection significantly downregulated FUT4 expression, whereas Anti-miR-200c transfection induced upregulation of FUT4 expression. These results were confirmed by cotransfection of miR-200c mimics and FUT4 cDNA (Figures 3e and k), as well as co-transfection of Anti-miR-200c and FUT4 siRNA (Figures $3 f$ and I). Immunofluorescent staining of FUT4 showed similar alterations (Figures $3 \mathrm{~g}$ and $\mathrm{m}$ ). The data indicates that miR-200c negatively regulates FUT4 expression by directly targeting its 3'-UTR of mRNA.

miR-200c suppresses $\alpha 1,3-f u c o s y l a t i o n$ on CD44 and inactivates $W n t / \beta$-catenin signaling pathway, further inhibits proliferation and adhesion. Because FUT4 is mainly responsible for adding fucose residue to the specific glycoproteins through the a1,3-linkage, and CD44 is a fucosylated glycoprotein that is highly expressed in the endometrial epithelium at the WOI, we explored whether miR-200c could regulate a1,3-fucosylation on CD44 by specifically targeting FUT4. We utilized Lotus tetragonolobus lectin (LTL) to detect the a1,3-fucosylated glycans of glycoproteins by lectin blot. The data showed that miR-200c mimics inhibited, whereas Anti-miR-200c promoted the biosynthesis of a1,3-fucosylated glycans on CD44 both in RL95-2 and Ishikawa cells. LeY is a difucosylated oligosaccharide that contains an a1,3-linkage fucose. The results also showed that miR-200c mimics decreased LeY biosynthesis; oppositely Anti-miR-200c increased LeY biosynthesis (Figures $4 \mathrm{a}$ and e). We further detected the alterations of CD44 fucosylation. Figures $4 \mathrm{~b}$ and $\mathrm{f}$ showed that downregulation of FUT4 by miR-200c mimics transfection inhibited a1,3-fucosylation on CD44, whereas upregulation of FUT4 by Anti-miR-200c enhanced a1,3-fucosylation, especially LeY biosynthesis on CD44. These results demonstrate the regulatory role of miR-200c on the biosynthesis of $a 1,3-$ fucosylation on CD44 (Figures 4b and f).

To clarify the signaling cascade underlying miR-200c and a1,3-fucosylation on CD44, the activation of Wnt/ $\beta$-catenin signaling pathway was detected. As shown in Figures $4 \mathrm{c}$ and $\mathrm{g}$, 
RL95-2

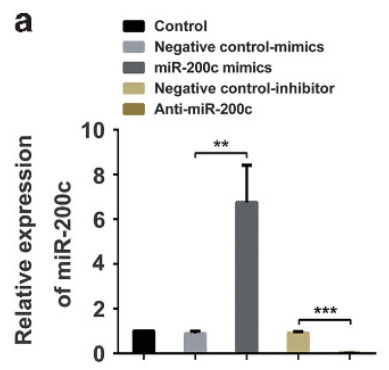

b $\rightarrow$ Control
- Negative control-mimics
- miR-200c mimics
$\rightarrow$ Negative control-inhibitor
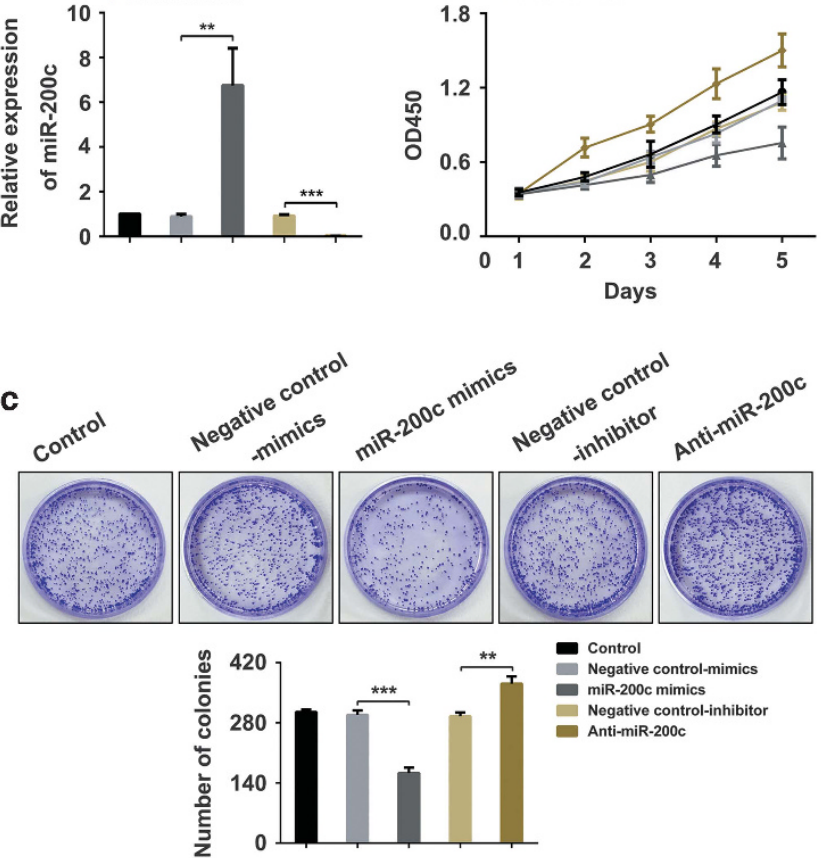

d
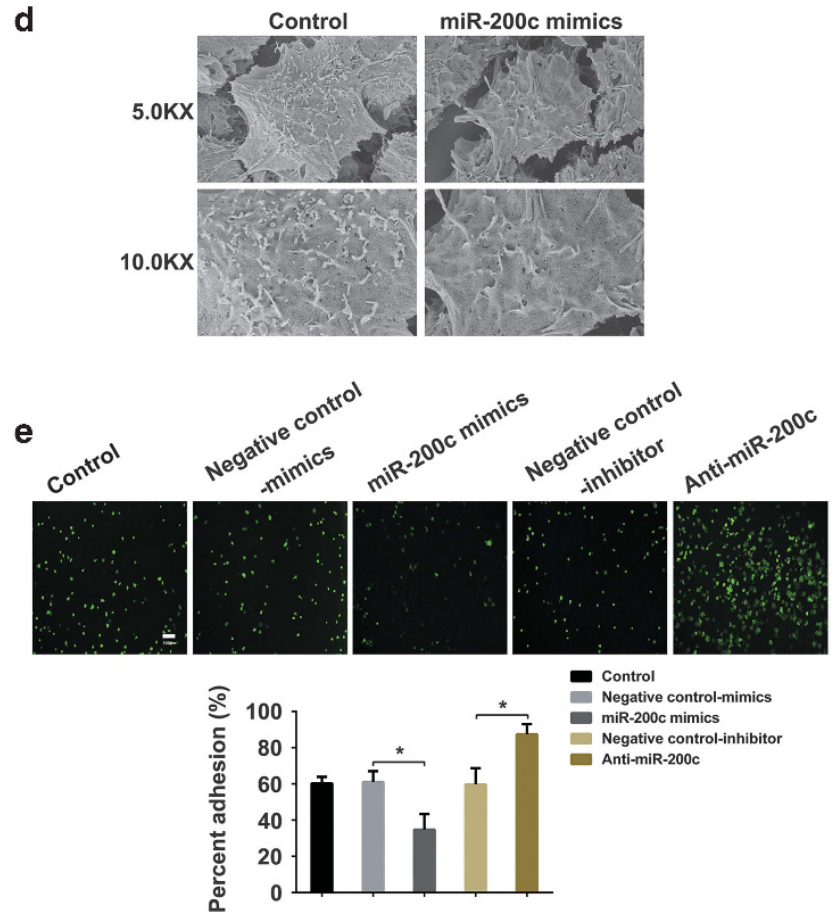

- Control

- Negative control-mimics

- miR-200c mimics

- Negative control-inhibitor
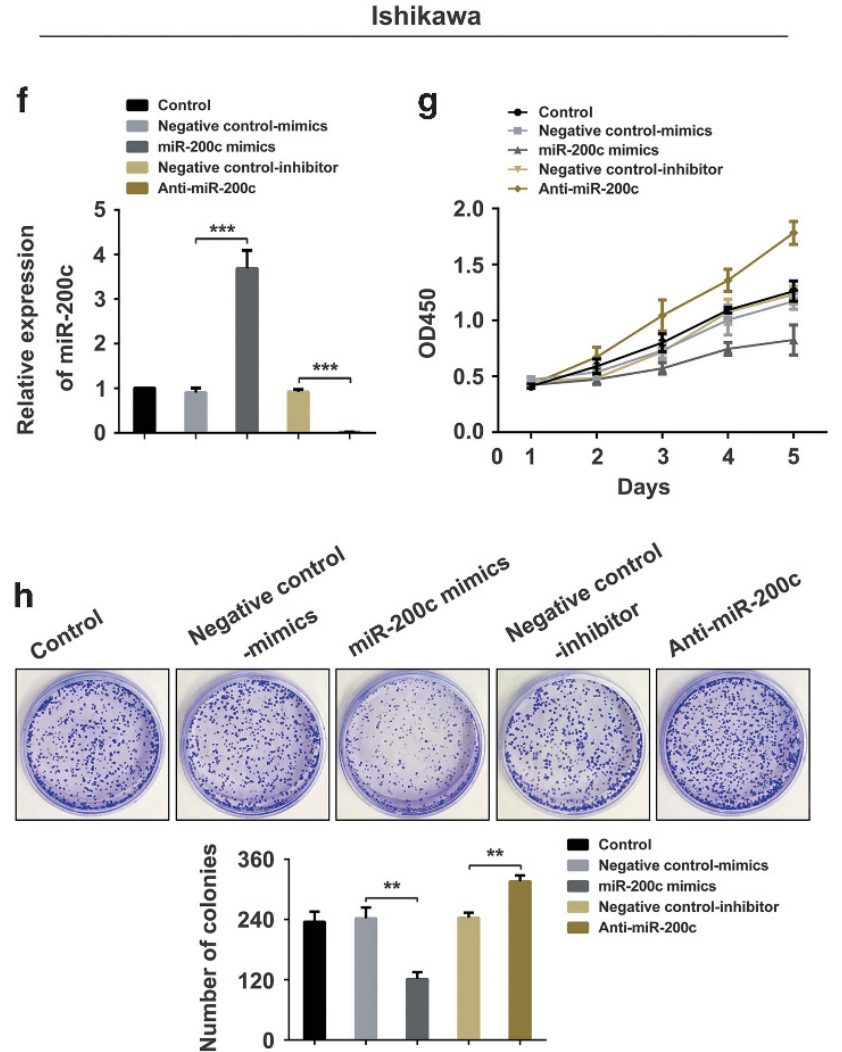

- Control

- Negative control-mimics miR-200c mimics Negative control-inhibitor Anti-miR-200c

i
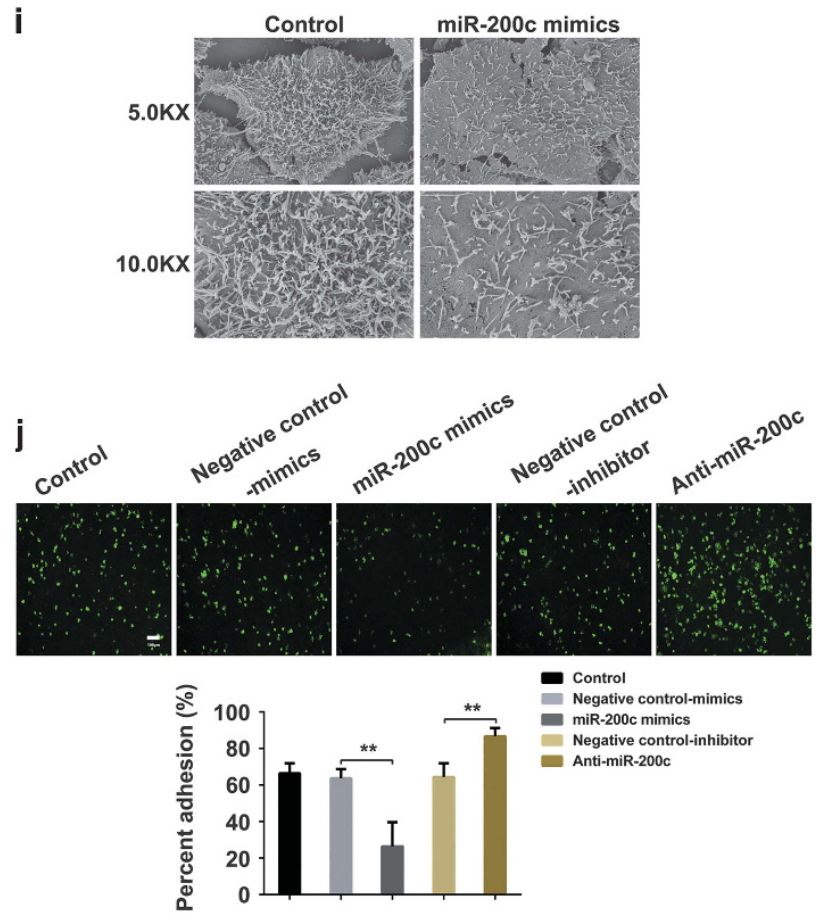

Figure 2 miR-200c inhibits proliferation and receptive ability of uterine epithelial cells in vitro. (a, f) Real-time PCR analysis for miR-200c expression in RL95-2 (a) and Ishikawa (f) cells after miR-200c mimics and Anti-miR-200c transfection. (b, $\mathbf{c}, \mathbf{g}, \mathbf{h})$ CCK-8 $(\mathbf{b}, \mathbf{g})$ and colony formation assay $(\mathbf{c}, \mathbf{h})$ were used to evaluate cell proliferation capabilities of RL95-2 and Ishikawa cells. (d, i) Representative pictures of scanning electron microscopy (SEM) in RL95-2 (d) and Ishikawa (i) cells after miR-200c mimics transfection. (e, j) Adhesion percent of embryonic cells (JAR) to RL95-2 (e) and Ishikawa (j) cells. The statistical analysis was shown. ${ }^{*} P<0.05,{ }^{* *} P<0.01,{ }^{* * *} P<0.001$ 
a

Wild type FUT4 3'UTR 5' TGA CTTAGgGGAGTT CAGTATT 3'

miR-200c 3' AgguaguaAuggGcCGUCAUAAU 5'

Mutant type FUT4 3'UTR 5' TGA CTT AGGgGAGT AATACTGAT 3'

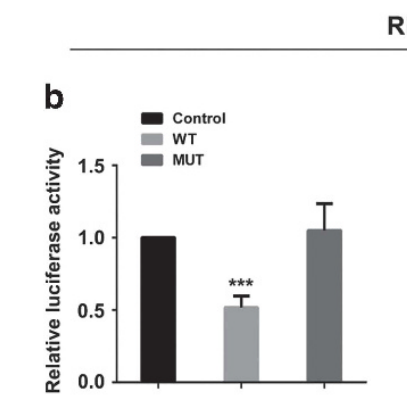

RL95-2

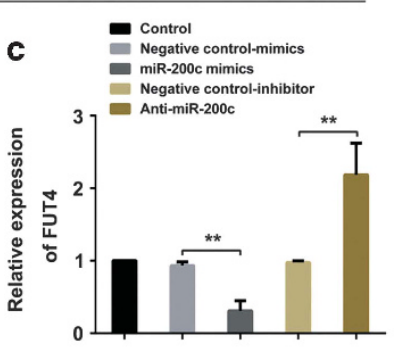

\section{d}
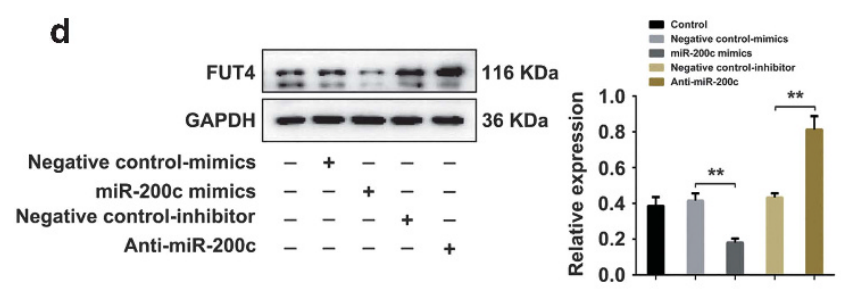

。
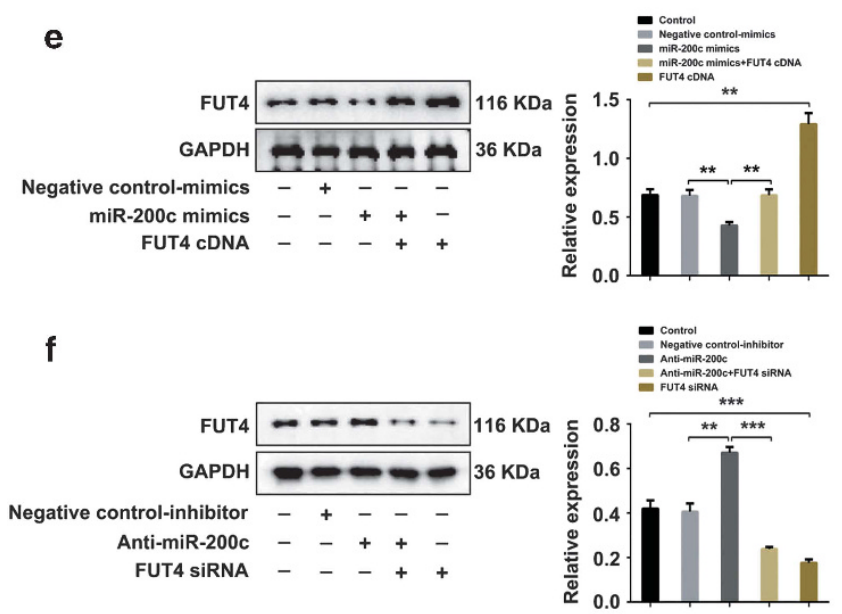

g
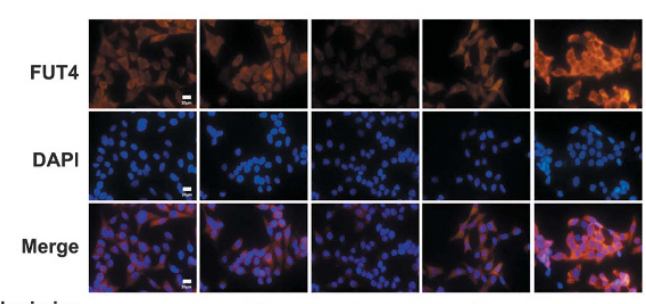

Negative control-mimics

miR-200c mimics

Negative control-inhibitor

Anti-miR-200c
$-$

-
-
$+$
$+$

Figure 3 FUT4 is a novel target of miR-200c. (a) Schematic representing reporter constructs of WT-FUT4 3'-UTR (upper panel), MUT-FUT4 3'-UTR with a mutated miR-200c binding site (lower panel) and miR-200c sequence (middle panel). (b, h) target of miR-200c was identified by dual-luciferase gene reporter assay in both RL95-2 (b) and lshikawa (h) cells. WT: wild-type FUT4 3'-UTR transfection; MUT: mutant-type FUT4 3'-UTR transfection. (c, d, i, j) Real-time PCR (c, i) and western blot analysis (d, j) of FUT4 level after miR-200c mimics and Anti-miR-200c transfection in uterine epithelial cells. The statistical analysis was shown. (e, k) Western blot analysis of FUT4 level in RL95-2 (e) and Ishikawa (k) cells transfected with miR-200c mimics or co-transfected with miR-200c mimics and FUT4 cDNA, respectively. The statistical analysis was shown. (f, I) Western blot analysis of FUT4 level in RL95-2 (f) and Ishikawa (I) cells transfected with Anti-miR-200c or co-transfected with Anti-miR-200c and FUT4 siRNA, respectively. The statistical analysis was shown. $(\mathbf{g}, \mathbf{m})$ Immunofluorescent staining of FUT4 after miR-200c mimics and Anti-miR-200c transfection in uterine epithelial cells. Scale bars, $20 \mu$ m. ${ }^{*} P<0.05$, ${ }^{* \star} P<0.01,{ }^{\star * \star} P<0.001$ 

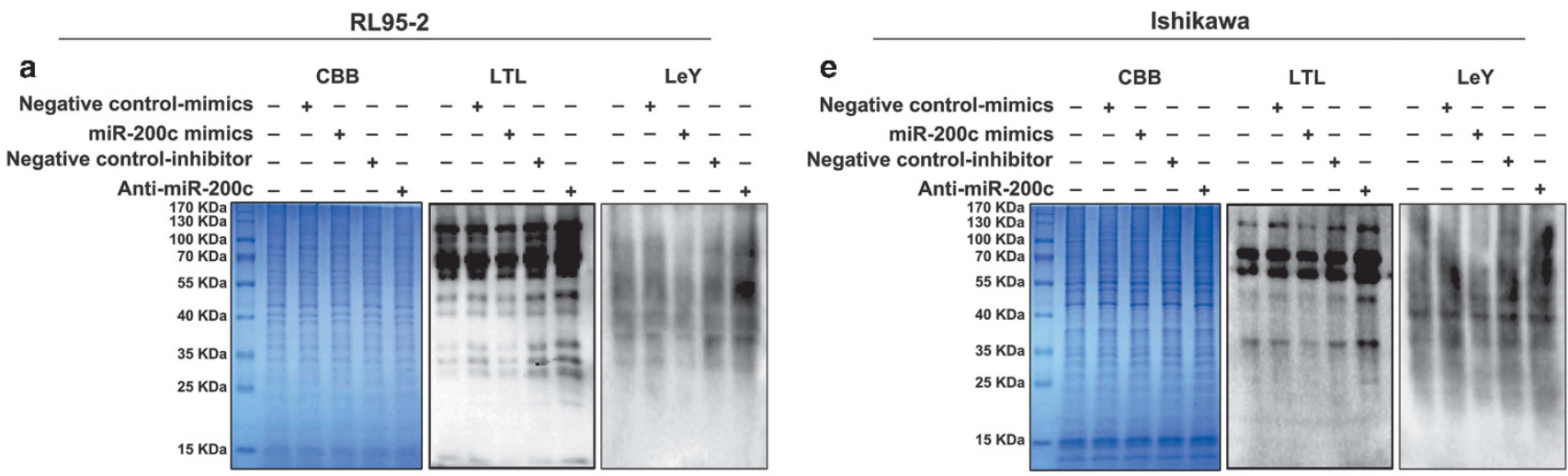

b

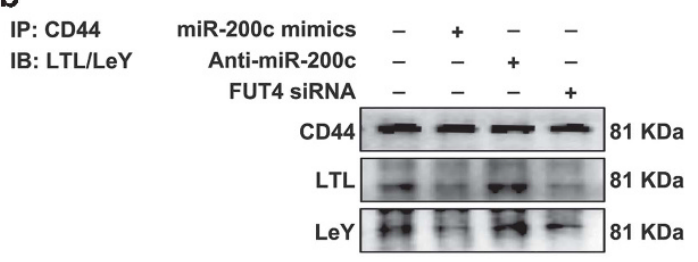

C

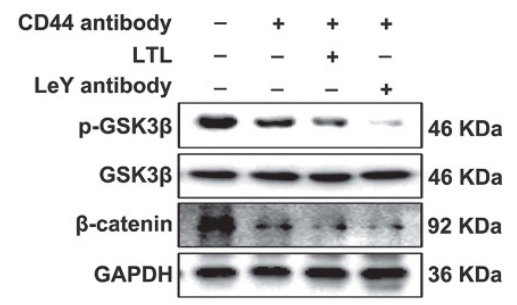

d

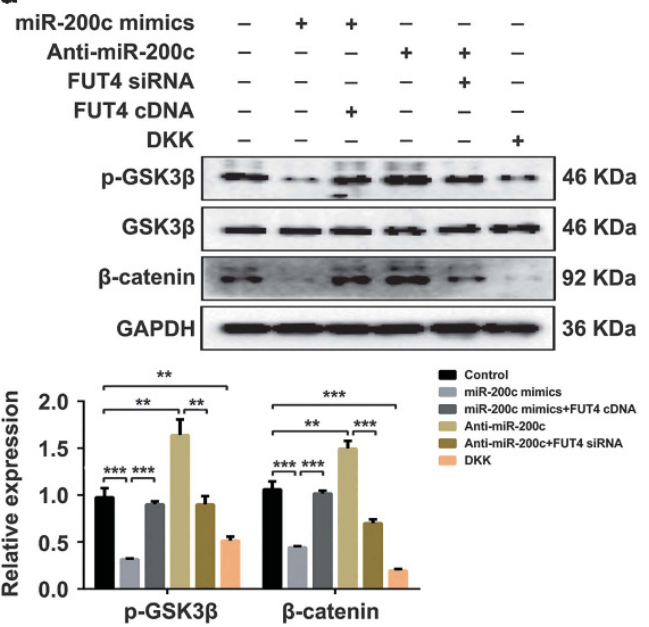

f

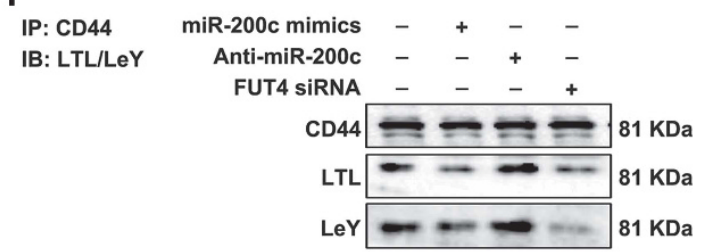

g

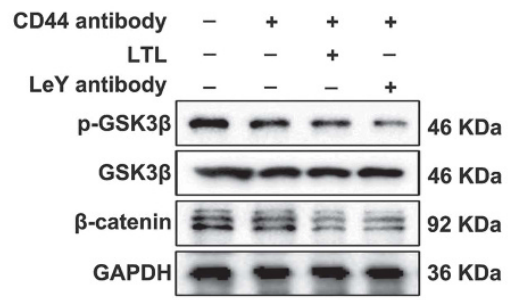

h

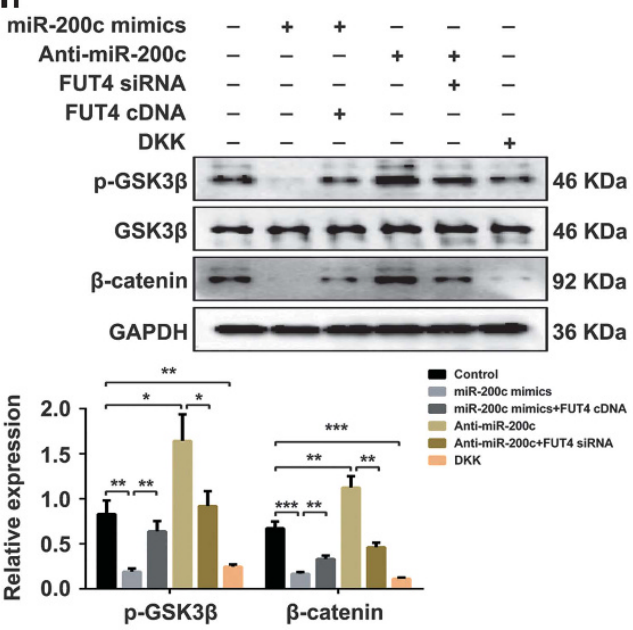

Figure 4 miR-200c decreases $\alpha 1$,3-fucosylation on CD44 and inactivates Wnt $/ \beta$-catenin signaling pathway. (a, e) Western/lectin blot analysis of effect of miR-200c on $\alpha 1,3$ fucosylation and LeY biosynthesis in RL95-2 (a) and Ishikawa (e) cells. CBB: coomassie brilliant blue. LTL: Lotus tetragonolobus lectin. (b, f) Immunoprecipitation and western blot analysis of $\alpha$ 1,3-fucosylation and LeY on CD44 in RL95-2 (b) and Ishikawa (f) cells. Immunoprecipitation (IP): anti-CD44 antibody pulled down protein. Immune blot (IB): detection of $\alpha$ 1,3-fucosylation by LTL lectin and anti-LeY antibody. (c, $\mathbf{g}$ ) Western blot analysis of CD44, LTL and LeY blocking on activation of p-GSK3 $\beta$, GSK3 $\beta$ and $\beta$-catenin in RL95-2 (c) and Ishikawa (g) cells. (d, h) Western blot and statistical analysis of p-GSK3 $\beta$, GSK3 $\beta$ and $\beta$-catenin in RL95-2 (d) and Ishikawa (h) cells. DKK: inhibitor of Wnt $\beta$ catenin signal pathway. ${ }^{*} P<0.05,{ }^{*} P<0.01, "{ }^{\prime \prime \prime} P<0.001$

anti-CD44 antibody blocking inactivated $\mathrm{Wnt} / \beta$-catenin signaling pathway, and LTL or anti-LeY antibody blocking strengthened the inactivation effect of CD44 on the $\mathrm{Wnt} / \beta$ catenin signaling pathway. Furthermore, p-GSK3 $\beta$ and $\beta$-catenin were also decreased after cells were transfected with miR-200c mimics, but partly recovered when cotransfected with miR-200c mimics and FUT4 cDNA. Moreover, Anti-miR-200c promoted the activation of $\mathrm{p}$-GSK3 $\beta$ and 
a

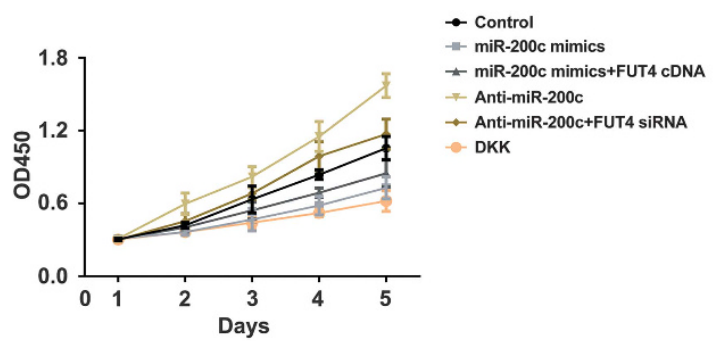

b
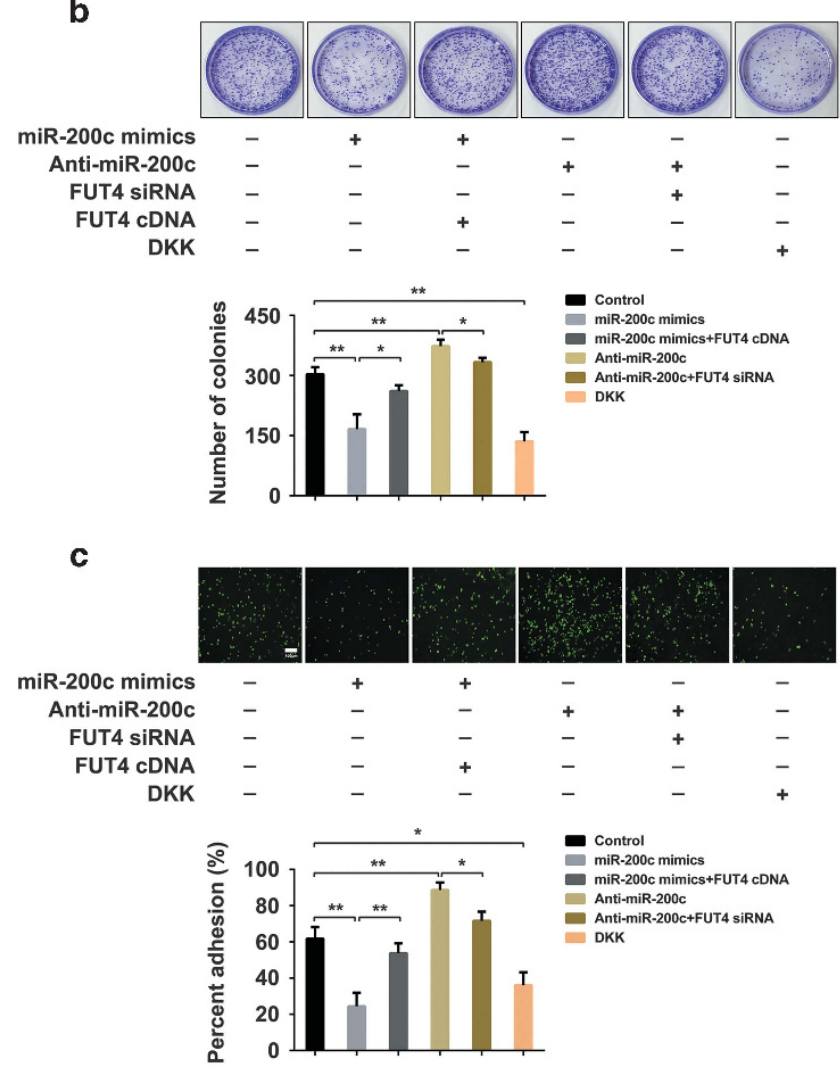

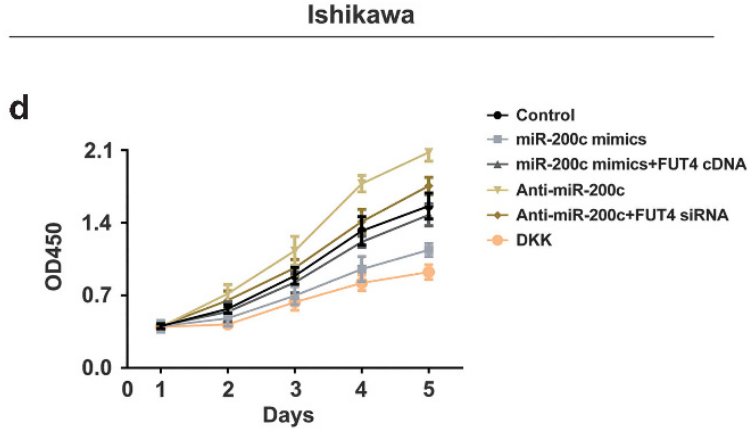

e

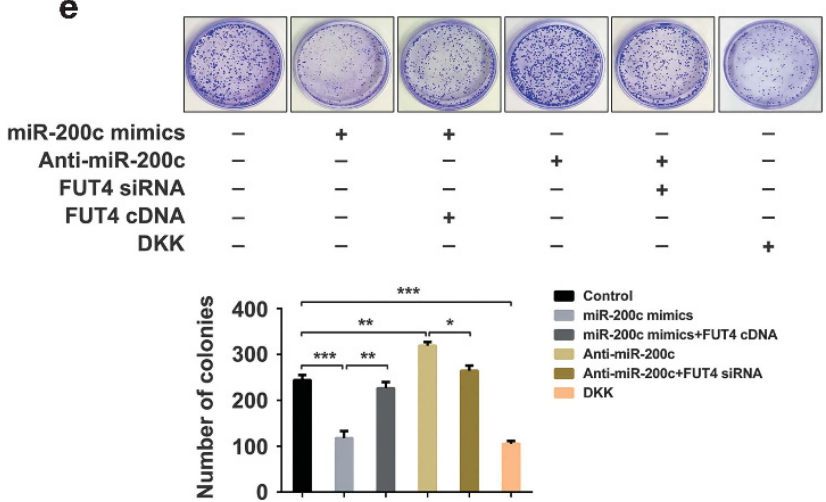

f

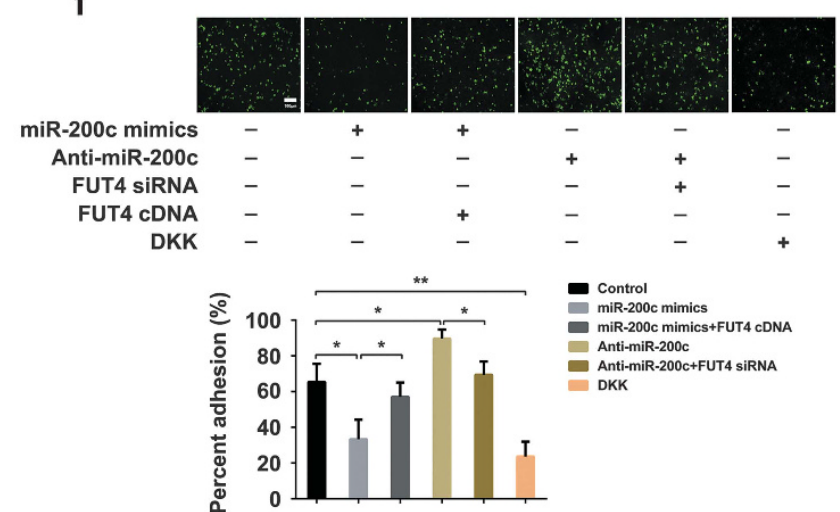

Figure 5 miR-200c inhibits proliferation and receptivity of uterine epithelial cells by targeting FUT4. (a, b, d, e) CCK-8 (a, d) and colony formation assay (b, e) were used to evaluate cell proliferation abilities of RL95-2 and Ishikawa cells. The statistical analysis was shown. (c, f) Adhesion percent of embryonic cells (JAR) to RL95-2 (c) and Ishikawa (f) cells. The statistical analysis was shown. ${ }^{*} P<0.05,{ }^{* *} P<0.01,{ }^{* * *} P<0.001$

$\beta$-catenin, but the reduced expression of $\mathrm{p}$-GSK3 $\beta$ and $\beta$ catenin were observed when co-transfection with AntimiR-200c and FUT4 siRNA in RL95-2 cells. Meantime, DKK, inhibitor of Wnt/ $\beta$-catenin signaling pathway, inactivated Wnt/ $\beta$-catenin signaling pathway (Figure $4 d$ ). Similar observations were achieved in Ishikawa cells (Figure 4h). The results indicate that the a1,3-fucosylation of glycoproteins, especially on CD44, can be suppressed by miR-200c mimics, and subsequently inactivate the downstream $\mathrm{Wnt} / \beta$-catenin signaling pathway.

We then determined whether the alteration in a1,3fucosylation by miR-200c and FUT4 was essential for uterine functions. The results showed that inactivated $\mathrm{Wnt} / \beta$-catenin signaling diminished the proliferation potential of endometrial epithelial cells by CCK-8 and colony formation assays, as well as adhesion percent between embryonic cells and endometrial cells by adhesion model in vitro. In contrast, activated Wnt/ $\beta$-catenin signaling by Anti-miR-200c increased cell proliferation ability and promoted embryonic cells adhered to endometrial RL95-2 and Ishikawa cells (Figure 5).

miR-200c inhibits uterine receptivity and embryo implantation in vivo. Having shown that miR-200c hampered proliferation and inhibited receptivity of uterine epithelial cells by targeting FUT4, we further investigated whether miR-200c inhibited uterine receptivity formation and subsequent embryo implantation in vivo. A mouse model was used to observe the role of miR-200c on uterine receptivity 
a

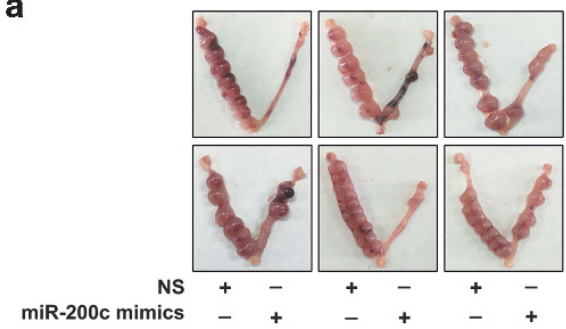

b

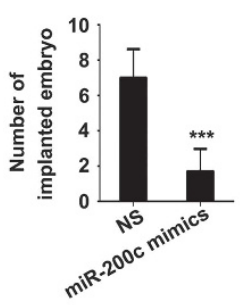

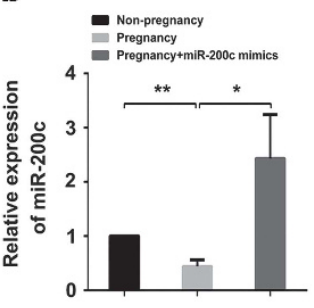

C

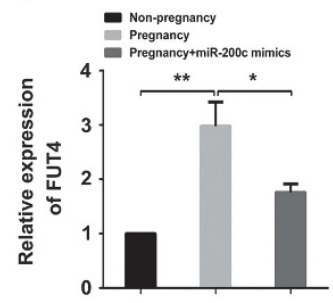

d

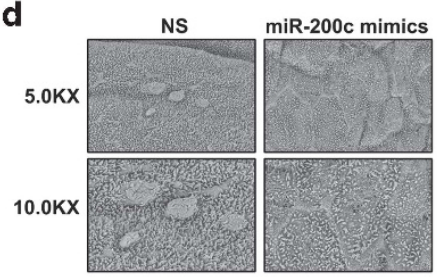

e

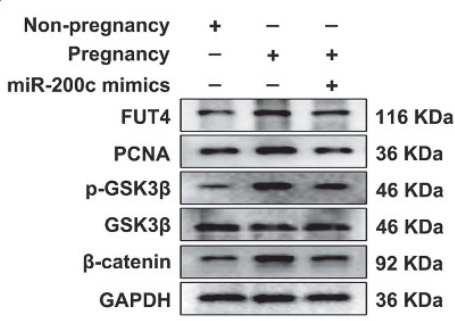

h

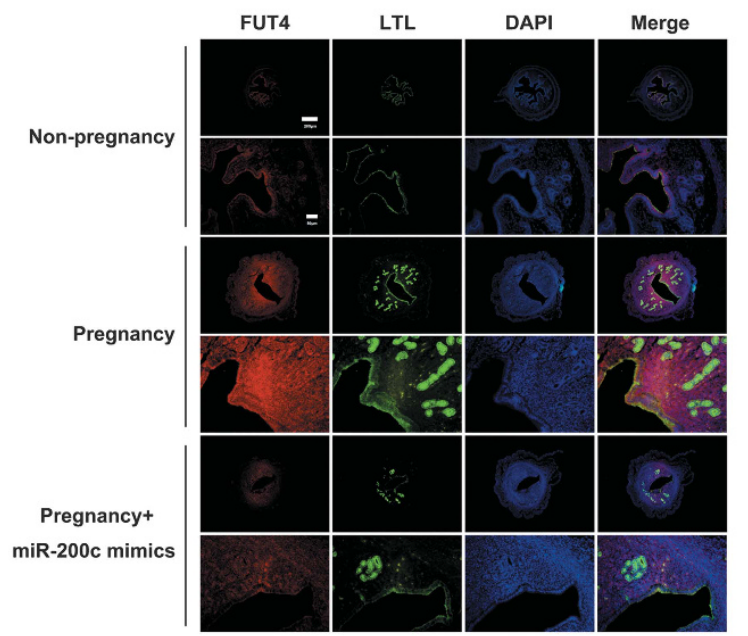

f $\frac{\text { CBB }}{+-- \text { LTL }}$ miR-200c mimics $-{ }_{-}+{ }_{-}+-{ }_{-}+$

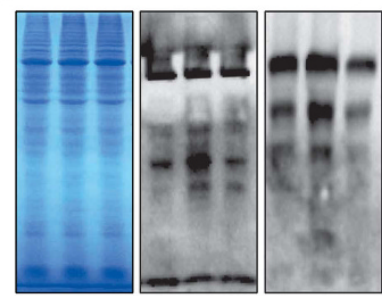$$
\text { (1) }
$$
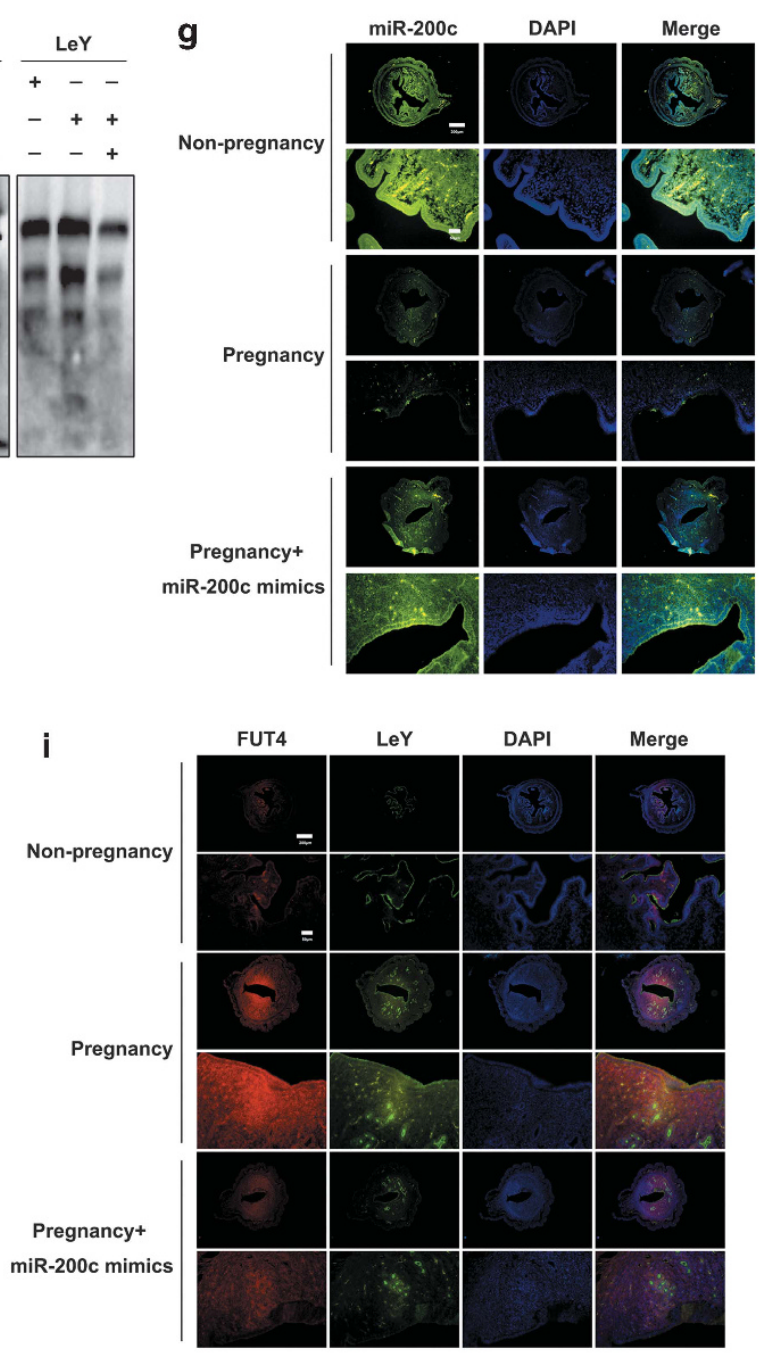

j

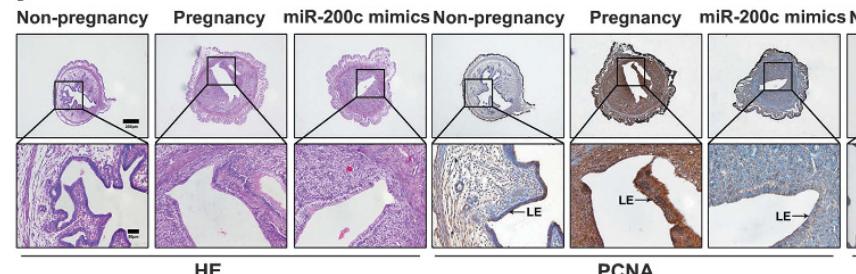

Pregnancy+

Pregnancy+

Pregnancy+

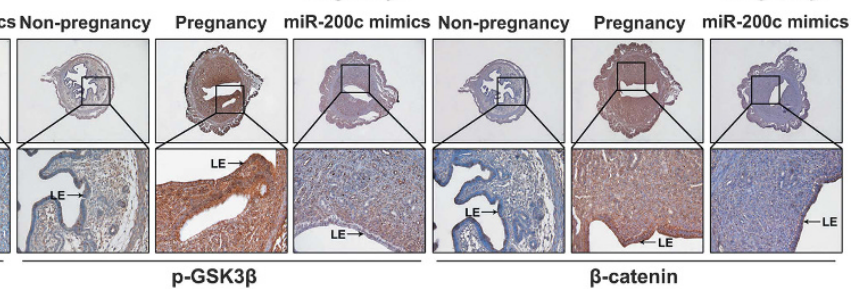

acquisition and embryo implantation. Injection of miR-200c mimics or normal saline into the mouse uterus was performed on day 3 of pregnancy, and the uterine receptivity was evaluated on day 4. As shown in Figure 6a, miR-200c significantly decreased embryo implantation rate in mouse. The decreased level of miR-200c was detected in the uterine endometrium of mouse on day 4 of pregnancy compared with non-pregnancy mouse by real-time PCR, while miR-200c 
Figure 6 miR-200c inhibits uterine receptivity formation and embryo implantation potential in vivo. Mouse uterus was injected with normal saline (NS) or miR-200c mimics on day 3 of pregnancy. (a) Number of implanted embryos in the uterus on day 9 pregnancy. The statistical analysis was shown. (b, c) Real-time PCR analysis of miR-200c (b) and FUT4 (c) in the uterine endometrium on day 4 of pregnancy. (d) Representative SEM images in the uterine endometrium after NS or miR-200c mimic injection on day 4 of pregnancy. (g) Fluorescent in situ hybridization (FISH) of miR-200c in the uterine endometrium on day 4 of pregnancy. (e, j) Western blot (e) and immunohistochemistry (j) analysis of PCNA and Wnt/ $\beta$-catenin signaling pathway in the uterine endometrium of pregnancy mouse. (f, h, i) Lectin blot (f) and immunofluorescenct staining (h, i) detection of $\alpha 1,3$ fucosylation (LTL), LeYand FUT4 in the uterine endometrium of pregnancy mouse. LE: luminal epithelium. The statistical analysis was shown. ${ }^{\star} P<0.05,{ }^{\star \star} P<0.01,{ }^{* \star *} P<0.001$

mimics injection increased miR-200c expression (Figure 6b). Meanwhile, FUT4 was significantly increased in the uterus of pregnant mouse, and the enhancing effect could be blocked by miR-200c mimics (Figures $6 \mathrm{c}$ and e). To further observe the expression and location of miR-200c in the uterine endometrium, fluorescence in situ hybridization (FISH) was conducted. As indicated in Figure 6g, weaker expression of miR-200c was observed in uterine endometrium of day 4 pregnant mouse, whereas stronger expression after miR-200c mimics injection.

To address the fact that miR-200c impaired uterine receptivity, the ultrastructure changes of uterine endometrium were also observed by SEM. The results revealed fewer microvilli and pinopode on the mouse uterus after miR-200c mimics injection than the control, implying the negative regulation of miR-200c on uterine receptivity formation (Figure 6d). PCNA, which is a proliferation marker, was increased in the uterus of pregnant mouse compared with in non-pregnancy mouse, whereas decreased in pregnancy mouse injected with miR-200c groups by western blot (Figure 6e) and immunohistochemistry (Figure 6j).

To confirm that the uterine receptivity was correlated with miR-200c and a1,3-fucosylation, we assessed the biosynthesis of $a 1,3$-fucosylated glycans and $\mathrm{Wnt} / \beta$-catenin signaling pathway activation in non-pregnancy, pregnancy and pregnancy injected with miR-200c mimics groups. The results showed that a1,3-fucosylation (LTL) and LeY epitope were mainly expressed on the uterine luminal and glandular epithelium, and reached a high level on day 4 of pregnancy, which was the WOI in mouse. However, miR-200c mimics significantly inhibited $a 1,3$-fucosylation and LeY biosynthesis (Figures $6 \mathrm{~h}$ and i), and the lectin blot results also showed the similar changes (Figure 6f). Meanwhile, Wnt/ $\beta$-catenin signaling pathway was activated in the pregnant uterus, whereas inactivated in the pregnant uterus injected with miR-200c by western blot and immunohistochemistry (Figures 6e and j).

\section{Discussion}

The establishment of uterine receptivity is a hallmark event for successful implantation of the embryo. Poor uterine receptivity correlates with infertility and abortion. ${ }^{31}$ In the present study, using clinical serum samples, cells, and mouse model, we demonstrated that the levels of the miR-200 family members, especially miR-200c, were significantly increased in the serum of the infertility patients compared with healthy non-pregnancy women. Meantime, the higher level of miR-200c in the abortion patients' serum was detected than normal pregnancy women. We also provided evidence that miR-200c inhibited uterine receptivity establishment by targeting FUT4, which inhibited the biosynthesis of $a-1,3$-fucosylation on glycoprotein CD44, and further inactivated $\mathrm{Wnt} / \beta$-catenin signaling pathway.
miRNAs have been found to be associated with the different functional states of uterine endometrium. Kresowik et al. ${ }^{32}$ revealed that 3 miRNAs (miR-30b, miR-30d and miR-31) were significantly elevated in the endometrial tissues of the secretory phase versus the proliferative phase. Estella et al. ${ }^{17}$ reported that a total of 26 miRNAs were upregulated, and 17 miRNAs were downregulated during human endometrial stromal decidualization in vitro, especially the downregulated miR-200 families. Ariel Revel et al. ${ }^{33}$ found that 10 miRNAs were high expression and 3 miRNAs low expression in the secretory endometrial tissues of patients who experienced repeated implantation failures compared with fertile women. It was also reported that miR-145 suppressed embryo-epithelial juxtacrine communication at implantation by targeting IGF1R in endometrial Ishikawa cells. ${ }^{34}$ In current study, we found that miR-200c level were lower in the human endometrial tissues of secretory phase than that in proliferative phase by FISH (Supplementary Figure 1). We also identified the lower level of miR-200c in mouse uteus of pregnant than non-pregnant mouse by FISH and real-time PCR. Furthermore, the serology analysis showed that the levels of miR-200 family members was remarkably increased in the serum of infertility patients compared with healthy women, and the levels was also higher in abortion patients than in normal pregnancy women. The sensitivity and specific analysis by ROC curve data suggests that miR-200c is a sensitive serum biomarker for evaluating endometrial receptivity. The in vitro cell experiments confirmed that the miR-200c mimics inhibited the receptive potential of epithelial cells. Therefore, miR-200c may be used as a potential new serum biomarker for infertility diagnosis and evaluation of endometrial receptivity.

The dynamic changes of the specific fucosyltransferases are closely correlated with the endometrial functions. The accumulated evidence shows that variations in the fucosyltransferase gene expression level play an important role in determining the implantation of the fetal-maternal interface. ${ }^{35}$ Overexpression of FUT7 in the uterus promoted embryo implantation in mouse. ${ }^{36}$ Ponnampalam et al revealed that FUT4 was significantly upregulated in the early- and mid-secretory phases of the menstrual cycle. ${ }^{23}$ Our previous results also demonstrated that cells with higher receptivity expressed an elevated level of FUT4, and overexpression of FUT4 in endometrial RL95-2 cells promoted embryonic cell adhesion. $^{24}$ The findings suggest that FUT4 is essential for establishing epithelial receptivity. Here, we further detected the serum level of FUT4 in different functional states, and found that FUT4 was decreased in infertility and abortion patients serum compared with healthy and pregnancy women. miRNAs alter target gene expression at the post-transcriptional level. Here, we verified that FUT4 regulated by miR-200c, could control the establishment of 
endometrium receptivity. In the present study, FUT4 was first predicted to be a direct target of $\mathrm{miR}-200 \mathrm{c}$ by a bioinformatics analysis, and was then confirmed by dual-luciferase reporter assays, real-time PCR and western blot. Our findings also demonstrated that the miRNA-200c mimic inhibited epithelial proliferation and receptivity by targeting FUT4. The above data manifestes that regulation of the specific fucosyltransferase by miRNA influences uterine receptive function.

Alterations in the fucosylation of glycoproteins greatly affect the cellular functions, including reproduction, immune and cancer metastasis and so on. ${ }^{37}$ Increased levels of fucosylation have been reported in a number of cancers. ${ }^{38}$ The elevated core fucosylation by FUT8 promoted epithelialmesenchymal transition in non-small cell lung cancer cells. ${ }^{39}$

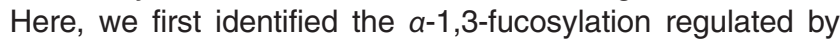
FUT4 changed the receptive potential of uterine endometrial cells to embryonic cells both in in vitro and in vivo. Our results showed that regulation of FUT4 expression by miR-200c mimics or Anti-miR-200c significantly decreased or increased the $a-1,3-$ fucosylation, respectively. Furthermore, for the first time, we reported that CD44, a receptive marker of endometrium, contains $a-1,3-$ fucosylation which directly influenced CD44 mediated endometrial receptive state. The fucosylated glycans of the glycoproteins on the uterine endometrium surface can recognize the corresponding ligands on the embryo through molecular recognition mediated by carbohydrate-carbohydrate interaction in embryo implantation. ${ }^{40,41}$ In this study, we found that CD44 contained LeY epitope in uterine epithelium, and the discovery of the specific binding ligands with CD44/LeY on embryo needs further study. CD44 exerts its functions by activating Wnt signaling pathway. ${ }^{42}$ Studies also manifested that $\mathrm{Wnt} / \beta$ catenin signaling pathway was involved in embryo implantation, stromal proliferation, deciduation and gland maintenance. ${ }^{43,44}$ Monhamed et al. ${ }^{45}$ reported that $\mathrm{Wnt} / \beta$ catenin signaling was first transiently activated in the uterine luminal epithelium at the prospective site of implantation in mouse. Here, we found that decreased LeY by miR-200c mimics transfection or LeY antibody blocking on CD44 inactivated $W n t / \beta$-catenin signaling pathway. The findings suggest that a tightly regulated miR-200c/FUT4/a-1,3-fucosylation(LeY)/CD44/Wnt/ $\beta$-catenin signaling cascade is essential for uterine receptivity.

In summary, we revealed that the miR-200 family members were increased in the serum of infertility patients compared with the non-pregnancy healthy women. Meantime, higher levels of miR-200 in the serum of abortion patients than normal early-pregnancy women. Moreover, we provided novel evidence showing that miR-200c inhibited $a-1,3-$ fucosylation and LeY carried on CD44 by targeting FUT4, which further inactivated $\mathrm{Wnt} / \beta$-catenin signaling pathway and caused receptive uterine endometrium formation failure. miR-200c and FUT4 may serve as the new biomarkers for the clinical diagnosis and strategy for infertility treatment.

\footnotetext{
Materials and Methods

Serum samples. Serum samples of healthy, infertility, early pregnancy or abortion women at the age of 25-35 were collected from The First Affiliated Hospital of Dalian Medical University (Dalian, China) between 2015 and 2016. The healthy controls (20 cases) were with regular menstrual cycle who were excluded from
}

other gynecological abnormalities. Infertility patients ( 20 cases) were defined by the failure to achieve a clinical pregnancy after 12 months, and excluded from pathological salpingemphraxis. The pregnant women (20 cases) were confirmed by ultrasound detection at $6-10$ gestational weeks. The abortion samples (20 cases) were from the first trimester (7-9 weeks) who underwent induced abortion. The samples were used to detect miR-200c and FUT4 levels. This study was approved by the Clinical Ethics Review Board of Dalian Medical University.

Cell culture. The human endometrial cell lines (RL95-2 and Ishikawa), and human embryonic JAR cell line were purchased from the American Type Culture Collection (Manassas, VA, USA). RL95-2 cells were maintained in DMEM/F12 (Invitrogen, Grand Island, NY, USA) supplemented with $10 \% \mathrm{FBS}, 0.005 \mathrm{mg} / \mathrm{ml}$ insulin (Sigma-Aldrich), $100 \mathrm{U} / \mathrm{ml}$ penicillin and $100 \mu \mathrm{g} / \mathrm{ml}$ streptomycin. Ishikawa and JAR cells were cultured in DMEM/F12 supplemented with $10 \% \mathrm{FBS}, 100 \mathrm{U} / \mathrm{ml}$ penicillin and $100 \mu \mathrm{g} / \mathrm{ml}$ streptomycin. Cells were maintained at $37^{\circ} \mathrm{C}$ under $5 \%$ $\mathrm{CO}_{2}$ in humidified incubator. The growth medium was changed every 2-3 days.

Transfection. miR-200c mimics (5'-UAAUACUGCCGGGUAAUGAUGGA-3'), Anti-miR-200c (5'-UCCAUCAUUACCCGGCAGUAUUA-3') and FUT4 siRNA (5'ACUCGAAGUUCAUCCAAACTT-3') were purchased from Genepharma (Shanghai, China). Cells were seeded into six-well plates prior to transfection. miR-200c mimics, Anti-miR-200c and FUT4 siRNA were transfected into cells when they reached 70\% confluence with lipofectamine 2000 reagent (Invitrogen) in accordance with the manufacturer's instructions. The miR-200c mimics and Anti-miR-200c were used at a final concentration of $100 \mathrm{nM}$ and FUT4 siRNA $50 \mathrm{nM}$, respectively. After transfection for $48 \mathrm{~h}$, RNA and protein samples were collected and real-time PCR, western blot were performed.

ELISA. ELISA kit (R\&D systems, Minneapolis, MN, USA) was used to detect the level of FUT4 in serum following the manufacturer's instructions. The absorbance at $450 \mathrm{~nm}$ was measured with a microplate reader (Thermo Fisher Scientific, Waltham, MA, USA) and the data were recorded. Three samples from each group were detected.

RNA isolation and real-time PCR. Total RNA from cells and tissues were extracted with Trizol reagent (Invitrogen). A PrimeScript RT Reagent Kit (TaKaRa, Dalian, China) was used for reverse transcription. Real-time PCR was performed with SYBR Premix Ex Taq II Kit (TaKaRa) and detected on ABI Prism 7500 Detection system (Applied Biosystem, Foster City, CA, USA). GAPDH and U6 was used as loading controls. Primers of miR-200c and U6 were obtained from Genepharma, and primers of FUT4 and GAPDH were from TaKaRa. Primer sequences were as followings: FUT4 (forward) 5 '-AAGGTCCAGGCCCACTGAAG -3', (reverse) 5'-CAGTTCAGGTGACAGAGGCTCAA-3'; GAPDH (forward) 5'ATGGGGAAGGTGAAGGTCG-3', (reverse) 5'-GGGGTCATTGATGGCAACAATA -3'. miR-200c (forward) 5'-GATCGTCATAATACTGCCG-3', (reverse) 5'-AGAGCA GGGTCCGAGGTA-3'; U6 (forward): 5'-ATTGGAACGATACAGAGAAGATT-3', (reverse): $5^{\prime}$-GGAACGCTTCACGAATTTG-3'. Each experiment was repeated at least three times.

Western blot. Cells were lysed in RIPA lysis buffer containing protease inhibitor cocktail (Roche, Basel, Switzerland). The protein concentration was determined with a BCA kit (Pierce, Rockford, IL, USA). Equal protein was separated with $12 \%$ SDSPAGE gel and electrotransferred onto nitrocellulose membranes (Millipore, Billerica, MA, USA). After blocking in $5 \%$ non-fat milk for $2 \mathrm{~h}$, the membranes were incubated with primary antibodies: FUT4 (1:1000, Proteintech, Wuhan, China), CD44 (1:1000, Abcam, Cambridge, UK), LTL (1:2000, Vector, California, USA), LeY (1:500, Abcam), p-GSK3 $\beta$ (1:500, Affinity Biosciences, Cincinnati, OH, USA), GSK3 $\beta$ (1:500, Affinity Biosciences), $\beta$-catenin (1:500, Affinity Biosciences), PCNA (1:100, Proteintech) and GAPDH (1:5000, Proteintech) at $4{ }^{\circ} \mathrm{C}$ overnight. After washing, the membranes were incubated with horseradish peroxidase-conjugated secondary antibody (1:3000) for $45 \mathrm{~min}$ at room temperature. The signals were visualized with $\mathrm{ECL}$ detection system. The relative protein level was quantified by densitometry and normalized to GAPDH level using Image J software (NIH, Bethesda, MD, USA).

Cell proliferation assay. Cell Counting Kit-8 (CCK-8, Dojindo Laboratories, Kumamoto, Japan) was used to assess the proliferation ability of RL95-2 and Ishikawa cells. Cells were plated into 96-well plates at a density of $3 \times 10^{3}$ cells per well and transfected with miR-200c mimics (50 nM), Anti-miR-200c (100 nM), FUT4 siRNA (50 nM) or FUT4 cDNA $(4 \mu \mathrm{g} / \mathrm{ml})$, respectively. CCK-8 solution was added to 
the medium and incubated at $37^{\circ} \mathrm{C}$ for $2 \mathrm{~h}$. Absorbance at $450 \mathrm{~nm}$ was measured using a microplate reader. The experiment was conducted in triplicate.

Colony formation assay. Cells were seeded into six-well plates with a density of $1 \times 10^{3}$ cells per well. The culture medium was subsequently changed every 2-3 days. miR-200c mimics, Anti-miR-200c, FUT4 siRNA or FUT4 cDNA were transfected into cells, respectively, with lipofectamine 2000 regent according to the protocols. After 2 weeks, the surviving colonies were fixed with methanol and stained with crystal violet. Images were captured under inverted microscope (Olympus, Tokyo, Japan). The colonies that contained $>50$ cells were counted. Data were recorded and statistically analyzed.

Cell adhesion assay. RL95-2 and Ishikawa cells were plated into 96-well plates to form a confluent monolayer. JAR cells $\left(1 \times 10^{4}\right)$ were first stained with Cell Tracker Green (CMFDA, Life Technologies, Carlsbad, CA, USA) for $1 \mathrm{~h}$. After staining, cells were harvested and suspended in $100 \mu \mathrm{l}$ of culture medium without FBS, and gently seeded onto the endometrial cell monolayer. After adhesion for $1 \mathrm{~h}$ at $37^{\circ} \mathrm{C}$, unattached JAR cells were removed with PBS washing, and an equal amount of unstained JAR cells were added into 3 blank wells. Adhesion rate was calculated as the percentage of attached JAR cells (e/b) after detection by multimode plate reader (PerkinElmer, Waltham, MA, USA). e: average fluorescence intensity value in each experiment group; $b$, average fluorescence intensity value in the blank wells. The images were taken with a fluorescence microscope (Olympus), and the representative images were shown.

Dual-luciferase gene reporter assay. The human 3'-UTR of FUT4 gene was cloned into pGL3 vector (GenePharma, Shanghai, China), and a mutant plasmid was constructed with site-directed mutagenesis. For luciferase assay, RL95-2 and Ishikawa cells were plated into 24-well plates, and co-transfected with $100 \mathrm{ng}$ of pGL3-FUT4 and $50 \mathrm{nM}$ of miR-200c mimics with lipofectamine 2000. After transfection for $24 \mathrm{~h}$, firefly luciferase activity and renilla luciferase activity was measured using the dual-luciferase reporter assay system (Promega, Madison, WI, USA).

Immunofluorescent assay. For immunofluorescent analysis, cells grown on the coverslips were fixed with $4 \%$ paraformaldehyde for 20 min followed by 3\% BSA (Beyotime, Shanghai, China) blocking for $1 \mathrm{~h}$ at room temperature. Cells were incubated with anti-FUT4 antibody $(1: 100)$ at $4{ }^{\circ} \mathrm{C}$ overnight. After washing with PBS for three times, the second antibody conjugated with TRITC (1:100, ZSGBBIO, Beijing, China) was used for $1 \mathrm{~h}$. The nucleus was counterstained with DAPI (1:1000, Beyotime) for $3 \mathrm{~min}$ and mounted in anti-fade solution (Beyotime) after washing. The images were captured with a fluorescent microscope, and the representative images were shown.

Immunohistochemistry. Uterine tissue sections slide $(4 \mu \mathrm{m})$ were incubated with xylol for deparafinized, and rehydrated in descending concentrations of ethanol following with antigen retrieval in citrate buffer ( $\mathrm{pH} \mathrm{6.0).} 0.3 \% \mathrm{H}_{2} \mathrm{O}_{2}$ was used to remove endogenous peroxidases by incubation for $10 \mathrm{~min}$ and blocking with $5 \%$ BSA for $45 \mathrm{~min}$ at room temperature. Primary antibodies: FUT4 (1:100), LTL (1:100), LeY (1:50), p-GSK3 $\beta$ (1:50), $\beta$-catenin (1:50) and PCNA (1:100) were applied at $4{ }^{\circ} \mathrm{C}$ overnight in a wet chamber. After washing with PBS, slides were incubated with second antibody for $45 \mathrm{~min}$ and visualized with diaminobenzide. The slides were counterstained with hematoxylin, and the evaluation was performed. Images were captured under an inverted microscope.

Immunoprecipitation. Immunoprecipitation was conducted with protein $A / G$ agarose beads (Thermo Fisher Scientific) according to the manufacturer's instructions. Cells after transfection were lysed and incubated in immunoprecipitation lysis buffer (Beyotime) for $10 \mathrm{~min}$ at room temperature. The extracts were incubated with anti-CD44-antibody $\left(2 \mu \mathrm{g} / \mathrm{ml}\right.$, Abcam) at $4{ }^{\circ} \mathrm{C}$ overnight, and the immunoprecipitates were purified by protein $A / G$ agarose beads with gentle rocking. The beads were washed for three times with extraction buffer and resuspended in $20 \mu$ l SDS loading buffer. The whole cell lysates and immunoprecipitates were incubated at $70{ }^{\circ} \mathrm{C}$ for $10 \mathrm{~min}$ followed with western blot analysis.

Scanning electron microscope. Cells and tissues were fixed in $4 \%$ paraformaldehyde and postixed in $2 \%$ osmium tetroxide. After washing, samples were dehydrated by a series of incubations in ethanol. Dehydration was continued by incubation in $95 \%$ ethanol, absolute ethanol and acetone. Surfaces of the cells and tissues were coated with gold and scanned with a SEM (S-3700 N, Hitachi, Tokyo, Japan).

Animal experiments. Kunming mice (6-8 weeks) were obtained from Animal Center of Dalian Medical University (Dalian, China). The procedures involved in the mouse studies were approved by the Institutional Review Board of Dalian Medical University. The mouse was maintained in controlled conditions $\left(22-25^{\circ} \mathrm{C}, 60 \%\right.$ humidity, 14 L:10D). Pregnant mouse was obtained by housing one female and one male mouse. The day found the vaginal plug was defined as gestation day 1 . On day 3 of pregnancy at 09:00, mouse was anesthetized and $10 \mu \mathrm{l}$ solution containing $1 \mu \mathrm{g}$ miR-200c mimics was injected into the right uterus horn while the left with normal saline. RNA and protein samples of mouse endometrium on day 4 and day 9 were collected. The number of implanted embryos was counted and analyzed statistically.

Fluorescence in situ hybridization. An in situ analysis was used to detect miR-200c level in the human endometrium of proliferative and secretary phases, and mouse endometrium in non-pregnancy, pregnancy and pregnancy injected with miR-200c mimics groups. The tissues were fixed with $4 \%$ paraformaldehyde overnight and embedded in paraffin for sections $(4 \mu \mathrm{m})$. The sections were deparaffinized following with proteinase $\mathrm{K}$ incubation $(20 \mu \mathrm{g} / \mathrm{ml})$ at $37^{\circ} \mathrm{C}$ for $20 \mathrm{~min}$. After washing, the sections were dehydrated and hybridized with biotin-labeled miR-200c probe (5'-TCCATCATTACCCGGCAGTATTA- ${ }^{\prime}$, GenePharma) and the scrambled control probe (5'-TTGTACTACACAAAAGTACTG-3', GenePharma) at $50 \mathrm{nM}$ at $37^{\circ} \mathrm{C}$ for $18 \mathrm{~h}$. Then, the slides were incubated with FITC-conjugated secondary antibody $\left(1: 100\right.$, BOSTER, Wuhan, China) at $37^{\circ} \mathrm{C}$ for $1 \mathrm{~h}$. The signals of miR-200c were detected under a fluorescent microscope, and representative images were shown.

Statistical analysis. The results were expressed as mean \pm S.E.M. Difference between groups was estimated with the Student's $t$-test. The Spearman correlation analysis was used to analyze the relationship between miR-200c and FUT4 in the serum. The receiver operating characteristic curve was applied to evaluate the diagnostic value of the miR-200 family members. The statistical significance was indicated as the follows: ${ }^{*} P<0.05,{ }^{* *} P<0.01$ and ${ }^{* * *} P<0.001$.

\section{Conflict of Interest}

The authors declare no conflict of interest.

Acknowledgements. This work was supported by the National Natural Science Foundation of China (No: 31670810 and 31770857), and National Natural Science Foundation of Liaoning (20170540240).

1. Su RW, Fazleabas AT. Implantation and establishment of pregnancy in human and nonhuman primates. Adv Anat Embryol Cell Biol 2015; 216: 189-213.

2. Sharma A, Kumar P. Understanding implantation window, a crucial phenomenon. J Hum Reprod Sci 2012; 5: 2-6.

3. Bartosch C, Lopes JM, Beires J, Sousa M. Human endometrium ultrastructure during the implantation window: a new perspective of the epithelium cell types. Reprod Sci 2011; 18: 525-539.

4. Salamonsen LA, Evans J, Nguyen HP, Edgell TA. The microenvironment of human Implantation: determinant of reproductive success. Am J Reprod Immunol 2016; 75: 218-225.

5. Riad ON, Hak AA. Assessment of endometrial receptivity using Doppler ultrasonography in infertile women undergoing intrauterine insemination. Gynecol Endocrinol 2014; 30: 70-73.

6. Germeyer A, Savaris RF, Jauckus J, Lessey B. Endometrial beta3 integrin profile reflects endometrial receptivity defects in women with unexplained recurrent pregnancy loss. Reprod Biol Endocrinol 2014; 12: 53.

7. Aghajanova L, Hamilton AE, Giudice LC. Uterine receptivity to human embryonic implantation: histology, biomarkers, and transcriptomics. Semin Cell Dev Biol 2008; 19: 204-211.

8. Bidarimath M, Khalaj K, Wessels JM, Tayade C. MicroRNAs, immune cells and pregnancy. Cell Mol Immunol 2014; 11: 538-547.

9. Rosenbluth EM, Shelton DN, Wells LM, Sparks AE, Van Voorhis BJ. Human embryos secrete microRNAs into culture media-a potential biomarker for implantation. Fertil Steril 2014; 101: 1493-1500.

10. Vilella F, Moreno-Moya JM, Balaguer N, Grasso A, Herrero M, Martínez S et al. HsamiR-30d, secreted by the human endometrium, is taken up by the pre-implantation embryo and might modify its transcriptome. Development 2015; 142: 3210-3221.

11. Zhang $Q$, Zhang $H$, Jiang $Y$, Xue $B$, Diao Z, Ding $L$ et al. MicroRNA-181a is involved in the regulation of human endometrial stromal cell decidualization by inhibiting Krüppel-like factor 12. Reprod Biol Endocrinol 2015; 13: 23. 
12. Sathyapalan T, David R, Gooderham NJ, Atkin SL. Increased expression of circulating miRNA-93 in women with polycystic ovary syndrome may represent a novel, non-invasive biomarker for diagnosis. Sci Rep 2015; 5: 16890.

13. Wang L, Huang W, Ren C, Zhao M, Jiang X, Fang X et al. Analysis of Serum microRNA Profile by Solexa Sequencing in Women With Endometriosis. Reprod Sci 2016; 23: 1359-1370.

14. Sandrim VC, Luizon MR, Palei AC, Tanus-Santos JE, Cavalli RC. Circulating microRNA expression profiles in pre-eclampsia: evidence of increased miR-885-5p levels. BJOG 2016 123: 2120-2128.

15. Liu W, Niu Z, Li Q, Pang RT, Chiu PC, Yeung WS. MicroRNA and embryo implantation. Am J Reprod Immunol 2016; 75: 263-271.

16. Jimenez PT, Mainigi MA, Word RA, Kraus WL, Mendelson CR. miR-200 regulates endometrial development during early pregnancy. Mol Endocrinol 2016; 30: 977-987.

17. Estella C, Herrer I, Moreno-Moya JM, Quiñonero A, Martínez S, Pellicer A et al. miRNA signature and Dicer requirement during human endometrial stromal decidualization in vitro. PLOS ONE 2012; 7: e41080.

18. Jasper MJ, Care AS, Sullivan B, Ingman WV, Aplin JD, Robertson SA. Macrophage- derived LIF and IL1B regulate alpha(1,2) fucosyltransferase 2 (Fut2) expression in mouse uterine epithelial cells during early pregnancy. Biol Reprod 2011; 84: 179-188.

19. Liu S, Wang J, Qin HM, Yan XM, Yang XS, Liu C et al. LIF upregulates poFUT1 expression and promotes trophoblast cell migration and invasion at the fetal-maternal interface. Cell Death Dis 2014; 5: e1396.

20. Engelstaedter V, Fluegel B, Kunze S, Mayr D, Friese K, Jeschke U et al. Expression of the carbohydrate tumour marker Sialyl Lewis A, Sialyl Lewis X, Lewis $Y$ and ThomsenFriedenreich antigen in normal squamous epithelium of the uterine cervix, cervical dysplasia and cervical cancer. Histol Histopathol 2012; 27: 507-514.

21. Ghosh D, Liu N, Zhu ZM, Sengupta J. Immunolocalization of Le(y) oligosaccharide in endometrium during menstrual cycle and effect of early luteal phase mifepristone administration on its expression in implantation stage endometrium of the rhesus monkey. Hum Reprod 1998; 13: 1374-1379.

22. Wang $X Q$, Zhu ZM, Fenderson BA, Zeng GQ, Cao YJ, Jiang GT. Effects of monoclonal antibody directed to LeY on implantation in the mouse. Mol Hum Reprod 1998; 4: 295-300.

23. Ponnampalam AP, Rogers PA. Expression and regulation of fucosyltransferase 4 in human endometrium. Reproduction 2008; 136: 117-123.

24. Liu S, Yang X, Wang J, Wei J, Zhang D, Wang X et al. Differential expression of LeY and fucosyltransferase IV correlates with the receptivity of RL95-2 and HEC-1 A human uterine epithelial cells. Cell Biol Int 2012; 36: 469-474.

25. Stowell SR, Ju T, Cummings RD. Protein glycosylation in cancer. Annu Rev Pathol 2015; 10 : 473-510.

26. Senbanjo LT, Chellaiah MA. CD44: a multifunctional cell surface adhesion receptor is a regulator of progression and metastasis of cancer cells. Front Cell Dev Biol 2017; 5: 18

27. Yaegashi N, Fujita N, Yajima A, Nakamura M. Menstrual cycle dependent expression of CD44 in normal human endometrium. Hum Pathol 1995; 26: 862-865.

28. Yurdakan G, Ekem TE, Bahadir B, Gun BD, Kuzey GM, Ozdamar SO. Expression of adhesion molecules in first trimester spontaneous abortions and their role in abortion pathogenesis. Acta Obstet Gynecol Scand 2008; 87: 775-782.

29. Knudtson JF, Tekmal RR, Santos MT, Binkley PA, Krishnegowda N, Valente P et al. Impaired development of early endometriotic lesions in CD44 knockout mice. Reprod Sci 2016; 23: $87-91$.

30. Lin WM, Karsten U, Goletz S, Cheng RC, Cao Y. Co-expression of CD173 (H2) and CD174 (Lewis $Y$ ) with CD44 suggests that fucosylated histo-blood group antigens are markers of breast cancer-initiating cells. Virchows Arch 2010; 456: 403-409.
31. Fatemi HM, Popovic-Todorovic B. Implantation in assisted reproduction: a look at endometrial receptivity. Reprod Biomed Online 2013; 27: 530-538.

32. Kresowik JD, Devor EJ, Van Voorhis BJ, Leslie KK. MicroRNA-31 is significantly elevated in both human endometrium and serum during the window of implantation: a potential biomarker for optimum receptivity. Biol Reprod 2014; 91: 17.

33. Revel A, Achache H, Stevens J, Smith $Y$, Reich R. MicroRNAs are associated with human embryo implantation defects. Hum Reprod 2011; 26: 2830-2340.

34. Kang YJ, Lees M, Matthews LC, Kimber SJ, Forbes K, Aplin JD. MiR-145 suppresses embryo-epithelial juxtacrine communication at implantation by modulating maternal IGF1R. J Cell Sci 2015; 128: 804-814.

35. Aplin JD, Jones CJ. Fucose, placental evolution and the glycocode. Glycobiology 2012; 22: 470-478.

36. Zhang Y, Liu S, Liu Y, Wang Z, Wang X, Yan Q. Overexpression of fucosyltransferase VII (FUT7) promotes embryo adhesion and implantation. Fertil Steril 2009; 91: 908-914.

37. Schneider M, Al-Shareffi E, Haltiwanger RS. Biological functions of fucose in mammals. Glycobiology 2017; 27: 601-618.

38. Miyoshi E, Moriwaki K, Nakagawa T. Biological function of fucosylation in cancer biology. J Biochem 2008; 143: 725-729.

39. Chen $\mathrm{CY}$, Jan YH, Juan YH, Yang CJ, Huang MS, Yu CJ et al. Fucosyltransferase 8 as a functional regulator of nonsmall cell lung cancer. Proc Natl Acad Sci USA 2013; 110: 630-635.

40. Eggens I, Fenderson BA, Toyokuni T, Hakomori S. A role of carbohydrate- carbohydrate interaction in the process of specific cell recognition during embryogenesis and organogenesis: a preliminary note. Biochem Biophys Res Commun 1989; 158: 913-920.

41. Zhu ZM, Kojima N, Stroud MR, Hakomori S, Fenderson BA. Monoclonal antibody directed to Le(y) oligosaccharide inhibits implantation in the mouse. Biol Reprod 1995; 52: 903-912.

42. Orian-Rousseau V. CD44 acts as a signaling platform controlling tumor progression and metastasis. Front Immunol 2015; 6: 154.

43. Hayashi K, Erikson DW, Tilford SA, Bany BM, Maclean JA 2nd, Rucker EB 3rd et al. Wnt genes in the mouse uterus: potential regulation of implantation. Biol Reprod 2009; 80: 989-1000.

44. Farah O, Biechele S, Rossant J, Dufort D. Porcupine-dependent Wnt signaling controls stromal proliferation and endometrial gland maintenance through the action of distinct WNTs. Dev Biol 2017; 422: 58-69.

45. Mohamed OA, Jonnaert M, Labelle-Dumais C, Kuroda K, Clarke HJ, Dufort D. Uterine Wnt/ beta-catenin signaling is required for implantation. Proc Natl Acad Sci USA 2005; 102 : 8579-8584.

(c) (i) (2)(2) This work is licensed under a Creative Commons Attribution-NonCommercial-ShareAlike 4.0 International License. The images or other third party material in this article are included in the article's Creative Commons license, unless indicated otherwise in the credit line; if the material is not included under the Creative Commons license, users will need to obtain permission from the license holder to reproduce the material. To view a copy of this license, visit http://creativecommons.org/licenses/by-nc-sa/4.0/

(C) The Author(s) 2017

Supplementary Information accompanies this paper on Cell Death and Differentiation website (http://www.nature.com/cdd) 